\title{
En bloc spondylectomy for spinal metastases: a review of techniques
}

\author{
Kevin C. Yao, M.D., Stefano Boriani, M.D., Ziya L. Gokaslan, M.D., \\ AND NaRaYan SundareSAN, M.D.
}

Mount Sinai Medical Center, New York, New York; Ospedale Maggiore, Instituto Rizzoli, Bologna, Italy; and The Johns Hopkins Hospital, Baltimore, Maryland

\begin{abstract}
Object. Spinal metastases are prevalent in the population of patients with cancer. Effective cancer therapy must incorporate treatment strategies for these lesions. Increasingly, surgery is being recognized as an effective treatment modality both for the patient's quality of life and potential oncological cure. En bloc spondylectomy is the surgical procedure of choice to obtain these goals. The purpose of this study was to examine critically the rationale, indications, and outcomes of en bloc spondylectomy for spinal metastases.

Methods. Outcomes in the authors' series of patients who underwent en bloc spondylectomy for spinal metastases are critically analyzed. The rationale and indications for this procedure are discussed. The Weinstein, Boriani, and Biagini surgical staging system for spinal tumors is described. A review of the literature is performed to examine further the rationale underlying this aggressive surgical approach to metastatic spinal disease.

Conclusions. En bloc spondylectomy is the treatment of choice for solitary and oligometastatic spinal metastases with biologically favorable histological findings. In appropriately selected patients, neurological outcome, pain control, and oncological control are significantly better after en bloc spondylectomy compared with radiation therapy. Oncological outcomes also exceed those of intralesional techniques. The Weinstein, Boriani, and Biagini surgical staging system provides a standard with which to plan surgical approaches and to compare surgical outcomes.
\end{abstract}

KEY WoRdS • metastasis • spine • resection • spondylectomy

Metastases to the skeleton are discovered in 60 to $70 \%$ of cancer patients at the time of death. Of these lesions, 40 to $50 \%$ are located within the spine. Effective cancer therapy must incorporate appropriate treatment of spinal metastases, both for the patient's quality of life and for oncological control. Surgery is an effective treatment modality in selected patients with spinal metastases. Several recent developments have facilitated an aggressive surgical approach to metastatic spinal disease. First, improvements in neuroimaging, especially magnetic resonance imaging, have provided more accurate and potentially earlier diagnosis of spinal involvement. Second, instrumentation provides safe and effective circumferential spinal reconstruction. Finally, oncologically oriented surgical procedures have evolved that provide the best chance for "cure" and local tumor control. In this paper, we discuss the indications, rationale, and results of these surgical procedures.

Abbreviations used in this paper: $\mathrm{VB}=$ vertebral body.

\section{EN BLOC EXCISION}

\section{Rationale for the Procedure}

Increasingly, patients with solitary sites of spinal involvement are being identified. It is now clearly established that patients with "bone-only" metastases from a variety of solid tumors have a considerably better prognosis than those with visceral metastases. ${ }^{21}$ This is particularly true in patients who present with a solitary site of relapse in the spine after a long disease-free interval. In addition to truly solitary lesions, patients with a single site of relapse in the spine with additional small foci of uptake, either adjacent or distant, are now classified as having "oligometastatic" disease. Patients with oligometastatic disease have low tumor burdens and can be considered part of the group of patients with solitary sites for treatment purposes. ${ }^{11}$ Several studies have shown that these patients with biologically favorable cancers and good performance status have the potential for long-term survival if the lesions are treated aggressively. This subset of pa- 
tients should be treated with the intent to "cure" rather than palliate. ${ }^{15}$

Of all the potentially curative strategies available, excision of the metastatic focus remains the only proven method of obtaining long-term palliation or cure for a variety of sites, including lung, liver, and brain. The value of this strategy for spinal sites is becoming increasingly important; our data and several other studies have shown that en bloc resection or spondylectomy can improve median survival times considerably and result in 5-year survival rates greater than $15 \% .^{17}$

Although there are a variety of newer therapeutic modalities such as intensity-modulated radiation therapy and stereotactic spinal radiosurgery that hold promise, it has not been shown that external-beam radiation therapy alone can achieve the goal of local control because of the limited tolerance of the spinal cord for conventional photon-beam radiation. To justify this surgical approach, the mortality rate should be minimal and surgical morbidity must be acceptably low. In our view, a skilled surgical team with experience and a multidisciplinary approach is required to obtain optimal outcomes. At present, our mortality rate related to spine surgery for tumor resection is less than $1 \%$, and morbidity is less than $10 \%$. Furthermore, we believe that the neurological morbidity associated with resection of spine tumors is minimal, apart from those instances in which a nerve root or dura mater has to be included as part of the resection for oncological purposes. ${ }^{1-5,7,8,10,12-14,16-18,20,22,23}$

Although the majority of spinal tumors involve the VB, other tumor patterns may also be observed. Purely posteriorly located lesions or those located laterally are candidates for en bloc resection. The goal of surgery undertaken for oncological purposes should be en bloc resection of the tumor, either in a "marginal" fashion or with wide margins. In some cases of small tumors involving only the $\mathrm{VB}$, wide resections can be accomplished using subtotal vertebrectomy alone via an anterior approach. In the majority of metastases in the thoracolumbar spine, complete en bloc resection generally requires spondylectomy.

\section{Patient Selection}

The goals of surgery in the treatment of metastatic disease are fivefold: 1) oncological cure; 2) spinal stabilization; 3) neurological palliation; 4) pain relief; and 5) histological diagnosis. In each patient, these goals must be evaluated in the context of the overall oncological prognosis, taking into account comorbid medical conditions that may obviate an aggressive surgical stance. For patients with solitary or oligometastatic disease in the spine without neurological deficit or spinal cord compression, the primary rationale for surgery is oncological, that is, to optimize the prospects of achieving local control, longterm palliation, or cure.

Because en bloc spondylectomy imposes considerable physiological stress due to the length of the procedure, the potential for considerable blood loss, and the frequent requirement of open thoracotomy, comorbid conditions should not be underestimated. Age and performance status are important variables in selecting candidates for surgery.

In the literature, a variety of prognostic scoring systems have been described that provide a reliable assessment of the potential survival time of patients with spinal metastatic disease. The best known is that described by Tokuhashi, et al., ${ }^{19}$ which has subsequently been validated and updated since the original report. These authors proposed six clinical and radiological parameters that include the following: 1) type of primary tumor; 2) patient's neurological grade; 3) general physical condition; 4) number of bone lesions; 5) number of visceral sites; and 6) number of spinal sites. More recently, Tokuhashi has modified this scoring system (unpublished data) to give the greatest weight to the primary tumor type, appropriately emphasizing that the nature of the primary tumor should be the most important factor in deciding whether surgery should be performed using "en bloc" or intralesional methods.

Tomita, et al., ${ }^{21}$ have also proposed a simpler scoring system to guide the spine surgeon in the choice of an appropriate surgical strategy for spinal metastases. Prognostic factors in Tomita's scoring system include the grade of the malignancy, extent of metastatic disease, and extent of bone metastasis. With this scoring system, these authors suggest that appropriate candidates for marginal or wide resection are either patients with slowly or moderately growing spinal metastases with limited, treatable visceral metastatic disease or patients harboring rapidly growing metastases isolated to one spinal location. In our recent report $^{17}$ of 80 patients with solitary spinal metastases who underwent gross-total resection of tumor by various procedures, the rate of locoregional recurrences for the entire series was $32 \%$, whereas only one $(17 \%)$ of six patients who underwent en bloc resection experienced a local recurrence. Although overall survival clearly depends on the tumor's histological features, the overall median survival time in this group of patients whose tumors exhibited various histological features was 30 months, with $18 \%$ surviving more than 5 years.

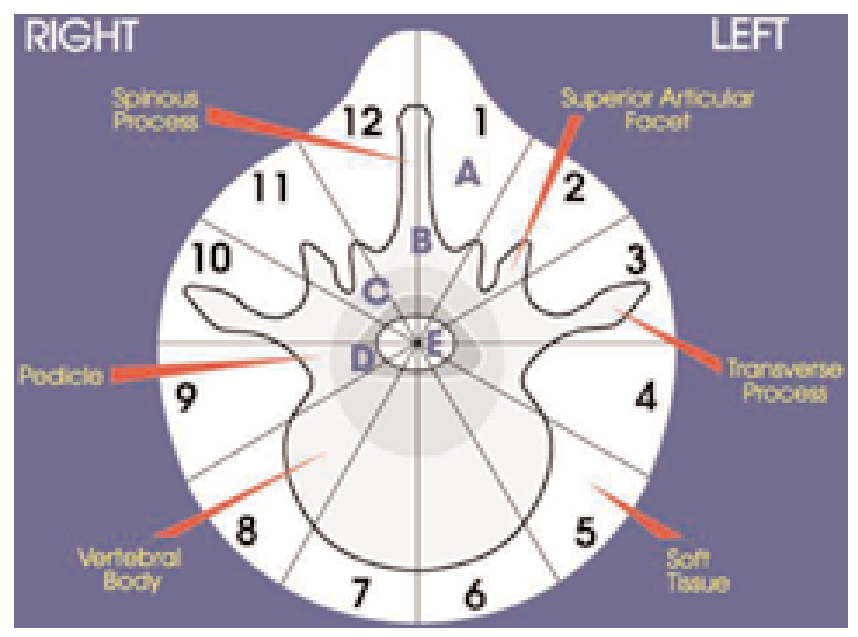

Fig. 1. Schematic drawing showing the Weinstein, Boriani, and Biagini surgical staging system. In this system, the transverse extension of vertebral tumors is described with reference to 12 radiating zones (numbered 1-12 in clockwise order) and five concentric layers (A-E, from the paravertebral extraosseous compartments to the dural involvement). The longitudinal extent of the tumor is recorded according to the levels involved. 


\section{Need for Surgical Staging}

After determining that the patient is an appropriate candidate for surgery by proper radiological staging of systemic disease, it is important to evaluate the local disease as completely as possible. The first attempt to propose a staging classification in the surgical management of spine tumors was introduced by Boriani, et al. ${ }^{6}$ (Fig. 1). We propose that this Weinstein, Boriani, and Biagini staging system be used in staging solitary spinal metastases as well as to provide a frame of reference for surgical planning and comparison of surgical outcomes.

\section{Description of Surgical Staging for Spine Tumors}

In the transverse plane, the vertebra is divided into 12 radiating zones (numbered 1-12 in a clockwise order) and five layers (A-E, from the paravertebral extraosseous region to the dural involvement). The longitudinal extent of the tumor is deduced by recording the spine segment(s) involved. It is our view that this system (Fig. 1) allows a more rational approach to surgical planning, provided that every effort is made to perform surgery along the required margins.

The major advantage of the clock-face radiating zone system is that it emphasizes the limitations of en bloc excision caused by the presence of the spinal cord in the longitudinal median axis of the vertebra. To save this vital structure and to control the epidural space (Layer D), the surgeon is compelled to resect wedge sectors of the vertebra. For example, if the tumor occupies an eccentrically shaped area, the surgeon must remove the healthy contralateral parts of the posterior arch, dissect the pseudocapsule from the dura, dislocate the dural sac, and create radiating sections by using a chisel or osteotome. There are three major methods for performing en bloc excisions in the thoracolumbar spine, depending on the location of the tumor: 1) vertebrectomy; 2) sagittal resection; and 3) resection of the posterior arch (Fig. 2). ${ }^{7}$

\section{DISCUSSION}

En bloc spondylectomy significantly decreases the rate of local recurrence and can provide long-term survival in a selected subset of patients with metastatic spinal disease. The senior authors of this paper (S.B., Z.L.G., N.S.) have performed spondylectomy for metastatic disease in more than 40 patients. Results from this series of patients are encouraging, with the mortality rate less than $1 \%$, morbidity less than $10 \%$, and median survival time longer than 3 years. These results validate those reported in smaller series. In the series published by Tomita, et al., ${ }^{21}$ 28 patients with metastatic spinal disease underwent wide or marginal excision, either by total en bloc spondylectomy (26 patients) or en bloc corpectomy (two patients). The mean length of survival in these patients was 38 months (range 6-84 months), with 93\% (26 patients) achieving local control and 32\% (nine patients) still alive at the last follow-up review.

Tumor violation by pediculotomy does not seem to correlate with the likelihood of tumor recurrence. In a series of 14 patients reported by Abe, et al., ${ }^{1}$ eight underwent total en bloc spondylectomy for solitary spinal metastasis. Meticulous histological analysis of the surgical margins in each resected vertebral specimen demonstrated no relationship between bisection of the specimen at a tumorinvolved pedicle and likelihood of local tumor recurrence. These authors, however, did report a 25\% local recurrence rate when attempting total spondylectomy via the posterior approach alone in patients with paravertebral spread of tumor anterior to the VB.

Resection of single metastatic sites of spinal cord compression offers the best neurological outcomes compared

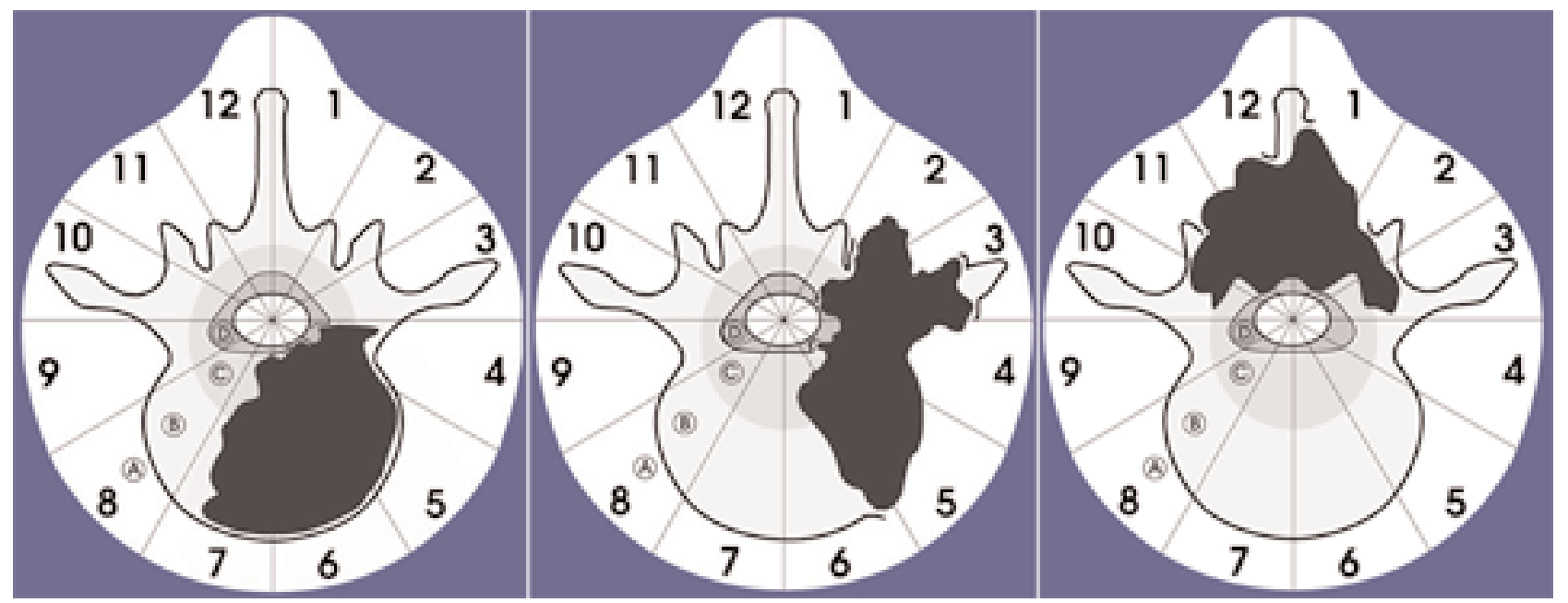

Fig. 2. Schematic drawings. Left: Drawing showing an indication for vertebrectomy. The en bloc excision of a lesion occurring in the VB can be performed with an oncologically appropriate margin if at least one pedicle is free of tumor. Center: Drawing showing an indication for sagittal resection. The en bloc excision of a tumor arising eccentrically in the body, the pedicle, or the transverse process is performed when the lesion occupies Zones 2 to 5 (or 8-11). Right: Drawing showing an indication for resection of the posterior arch. The en bloc excision of a tumor arising in the arch is performed when the lesion occupies Zones 10 to 3. The pedicles must be free of tumor to obtain an oncologically appropriate specimen. 
with other treatment modalities. In the series of 28 patients with metastatic spinal disease who underwent wide or marginal excision reported by Tomita, et al., ${ }^{21} 18$ (64\%) presented with neurological compromise. After surgery, $14(78 \%)$ of these 18 patients demonstrated improvement. Abe, et al., ${ }^{1}$ reported at least a one-grade improvement on the modified Frankel scale in all eight patients in their series who underwent total en bloc spondylectomy for solitary spinal metastasis. In our series of 80 patients who underwent aggressive resection of solitary metastases, 48\% improved at least one Frankel grade, $45 \%$ remained neurologically intact, $6 \%$ remained neurologically impaired but unchanged, and one patient worsened. ${ }^{17} \mathrm{Sim}-$ ilarly, Patchell's report ${ }^{14}$ included 32 patients who entered the study unable to walk. Of these, nine $(56 \%)$ of 16 patients who underwent surgery followed by radiation therapy regained the ability to walk, compared with only three $(19 \%)$ of 16 patients treated with radiation alone. In fact, three $(30 \%)$ of 10 patients who experienced neurological worsening while receiving radiation therapy regained the ability to walk after subsequent surgery. Surgically treated patients also maintained continence and functional Frankel and American Spinal Injury Association Scale scores significantly longer than patients in the radiation group.

Total en bloc spondylectomy is also effective at controlling spinal pain. Abe, et al., ${ }^{1}$ reported relief of intractable spinal pain in all eight of their patients who underwent total en bloc spondylectomy for solitary spinal metastasis. Similarly, we reported a $95 \%$ rate of pain improvement in patients with solitary spinal metastases, with complete relief in $76 \% .{ }^{17}$ Patchell ${ }^{14}$ also reported significantly reduced narcotic use after resection and irradiation of spinal metastases compared with radiation treatment alone.

Patients with metastatic spinal disease are subject to a host of comorbidities associated with the underlying malignancy and its treatment, as well as the morbidities associated with a neurologically compromised state. Patients may be immunosuppressed, nutritionally depleted, and immobilized. In a retrospective study examining the rate of complications after surgery for spinal metastasis, Wise, et al., ${ }^{23}$ reported complications in $25 \%$ of the procedures, with major complications occurring in $13 \%$. The most common major complications after surgery for spinal metastases were postoperative wound infection, pneumonia, pulmonary embolism, and hardware failure. Although no patient died because of an intraoperative event, the mortality rate in their series was $5 \%$, with deaths most often caused by postoperative sepsis. Poor preoperative neurological function (based on either the Harrington classification or Frankel grade) predicted a higher occurrence rate of both major and minor complications. Preoperative radiation therapy also correlated significantly with a higher complication rate.

These results agree with our data showing that preoperative radiation therapy given to patients with solitary spinal metastases contributes significantly to postoperative complications, especially wound complications. ${ }^{17}$ In our study, complications developed in six (15\%) of 40 patients who underwent de novo surgery, compared with 16 $(40 \%)$ of 40 patients who received prior radiation. Ghogawala, et al., ${ }^{9}$ reported a major wound complication rate of $12 \%$ in patients who underwent de novo surgery, compared with $32 \%$ in patients who had previously received radiation therapy. Patchell's series ${ }^{14}$ included a $12 \%$ rate of surgical complications after de novo surgery, compared with $40 \%$ in patients who had previously received radiation therapy. These rates of surgical morbidity are acceptable risks in the management of spinal metastases, particularly because combined treatment (surgery followed by radiation therapy) is clearly superior to radiation therapy alone in virtually all patients with spinal metastatic disease, regardless of whether the spinal lesion is solitary.

\section{CONCLUSIONS}

En bloc spondylectomy is the treatment of choice for solitary and oligometastatic spinal metastases with biologically favorable histological findings. In appropriately selected patients, neurological outcome, pain control, and oncological control are significantly better after en bloc spondylectomy compared with radiation therapy. Oncological outcomes also exceed those of intralesional techniques. The Weinstein, Boriani, and Biagini surgical staging system provides a standard with which to plan surgical approaches and to compare surgical outcomes.

\section{References}

1. Abe E, Kobayashi T, Murai H, et al: Total spondylectomy for primary malignant, aggressive benign, and solitary metastatic bone tumors of the thoracolumbar spine. J Spinal Disord 14: 237-246, 2001

2. Biagini R, Boriani S, Andreoli I, et al: Surgical technique: dorsal vertebral hemiresection for bone tumors. Chir Organi Mov 79:331-337, 1994

3. Boriani S, Biagini R, Andreoli I, et al: Resection of the vertebral arch in the treatment of neoplasms of the spine. Chir Organi Mov 80:183-189, 1995

4. Boriani S, Biagini R, De Iure F, et al: En bloc resections of bone tumors of the thoracolumbar spine. A preliminary report on 29 patients. Spine 21:1927-1931, 1996

5. Boriani S, Biagini R, De Iure F, et al: Lumbar vertebrectomy for the treatment of bone tumors: surgical technique. Chir Organi Mov 79:163-173, 1994

6. Boriani S, Weinstein JN, Biagini R: Primary bone tumors of the spine. Terminology and surgical staging. Spine 22:1036-1044, 1997

7. Fourney DR, Abi-Said D, Lang FF, et al: Use of pedicle screw fixation in the management of malignant spinal disease: experience in 100 consecutive procedures. J Neurosurg (Spine 1) 94: 25-37, 2001

8. Fourney DR, Abi-Said D, Rhines LD, et al: Simultaneous anterior-posterior approach to the thoracic and lumbar spine for the radical resection of tumors followed by reconstruction and stabilization. J Neurosurg (Spine 2) 94:232-244, 2001

9. Ghogawala Z, Mansfield FL, Borges LF: Spinal radiation before surgical decompression adversely affects outcomes of surgery for symptomatic metastatic spinal cord compression. Spine 26:818-824, 2001

10. Gokaslan ZL, York JE, Walsh GL, et al: Transthoracic vertebrectomy for metastatic spinal tumors. J Neurosurg 89: 599-609, 1998

11. Hortobagyi GN: Can we cure limited metastatic breast cancer? J Clin Oncol 20:620-623, 2002 
12. Kavolius JP, Mastorakos DP, Pavlovich C, et al: Resection of metastatic renal cell carcinoma. J Clin Oncol 16:2261-2266, 1998

13. Pascal-Moussellard H, Broc G, Pointillart V, et al: Complications of vertebral metastasis surgery. Eur Spine J 7: 438-444, 1998

14. Patchell R, Tibbs PA, Regine WF, et al: A randomized trial of direct decompressive surgical resection in the treatment of spinal cord compression caused by metastasis. Proc Am Soc Clin Oncol 22:1, 2003 (Abstract)

15. Singletary SE, Walsh G, Vauthey JN, et al: A role for curative surgery in the treatment of selected patients with metastatic breast cancer. Oncologist 8:241-251, 2003

16. Sundaresan N, DiGiacinto GV, Krol G, et al: Spondylectomy for malignant tumors of the spine. J Clin Oncol 7:1485-1491, 1989

17. Sundaresan N, Rothman A, Manhart K, et al: Surgery for solitary metastases of the spine: rationale and results of treatment. Spine 27:1802-1806, 2002

18. Sundaresan N, Steinberger AA, Moore F, et al: Indications and results of combined anterior-posterior approaches for spine tumor surgery. J Neurosurg 85:438-446, 1996
19. Tokuhashi Y, Matsuzaki H, Toriyama S, et al: Scoring system for the preoperative evaluation of metastatic spine tumor prognosis. Spine 15:1110-1113, 1990

20. Tomita K, Kawahara N, Baba H, et al: Total en bloc spondylectomy for solitary spinal metastasis. Int Orthop 18:291-298, 1994

21. Tomita K, Kawahara N, Kobayashi T, et al: Surgical strategy for spinal metastases. Spine 26:298-306, 2001

22. Weigel B, Maghsudi M, Neumann C, et al: Surgical management of symptomatic spinal metastases. Postoperative outcome and quality of life. Spine 24:2240-2246, 1999

23. Wise JJ, Fischgrund JS, Herkowitz HN, et al: Complication, survival rates, and risk factors of surgery for metastatic disease of the spine. Spine 24:1943-1951, 1999

Manuscript received September 24, 2003.

Accepted in final form October 10, 2003.

Address reprint requests to: Kevin C. Yao, M.D., Department of Neurosurgery, M. D. Anderson Cancer Center, University of Texas, Box 442, 1515 Holcombe Boulevard, Houston, Texas 77030. email: kyao@mdanderson.org. 
Author Names, et al. 